\title{
Terahertz-Driven Nonlinear Spin Response of Antiferromagnetic Nickel Oxide
}

\author{
S. Baierl, ${ }^{1}$ J. H. Mentink, ${ }^{2}$ M. Hohenleutner, ${ }^{1}$ L. Braun, ${ }^{3}$ T.-M. Do, ${ }^{1}$ C. Lange, ${ }^{1}$ \\ A. Sell, ${ }^{4}$ M. Fiebig, ${ }^{5}$ G. Woltersdorf, ${ }^{6}$ T. Kampfrath, ${ }^{3}$ and R. Huber ${ }^{1, *}$ \\ ${ }^{1}$ Department of Physics, University of Regensburg, 93053 Regensburg, Germany \\ ${ }^{2}$ Radboud University, Institute of Molecules and Materials, Heyendaalseweg 135, 6525 AJ Nijmegen, Netherlands \\ ${ }^{3}$ Department of Physical Chemistry, Fritz. Haber Institute of the Max Planck Society, Faradayweg 4-6, 14195 Berlin, Germany \\ ${ }^{4}$ Department of Physics, University of Konstanz and TOPTICA Photonics AG, Lochhamer Schlag 19, 82166 Gräfelfing, Germany \\ ${ }^{5}$ Department of Materials, ETH Zurich, Vladimir-Prelog-Weg 4, 8093 Zurich, Switzerland \\ ${ }^{6}$ Institute of Physics, Martin Luther University Halle-Wittenberg, 06120 Halle, Germany \\ (Received 25 September 2015; revised manuscript received 26 April 2016; published 1 November 2016)
}

\begin{abstract}
Terahertz magnetic fields with amplitudes of up to 0.4 Tesla drive magnon resonances in nickel oxide while the induced dynamics is recorded by femtosecond magneto-optical probing. We observe distinct spin-mediated optical nonlinearities, including oscillations at the second harmonic of the $1 \mathrm{THz}$ magnon mode. The latter originate from coherent dynamics of the longitudinal component of the antiferromagnetic order parameter, which are probed by magneto-optical effects of second order in the spin deflection. These observations allow us to dynamically disentangle electronic from lattice-related contributions to magnetic linear birefringence and dichroism - information so far only accessible by ultrafast THz spin control. The nonlinearities discussed here foreshadow physics that will become essential in future subcycle spin switching.
\end{abstract}

DOI: 10.1103/PhysRevLett.117.197201

Selectively controlling the electron spin on ultrashort time scales has become a key challenge of condensed-matter physics. Achieving this goal is expected to reveal fascinating fundamental many-body physics and to enable disruptive technology for information processing at optical clock rates [1-27]. A variety of promising magneto-optical control schemes has been developed in recent years, including the inverse Faraday effect and Raman-type nonlinear optical processes $[2,4,5,7-14]$ as well as optical modification of the exchange interaction [15-17]. Although these techniques have revolutionized our understanding of ultrafast spin dynamics, the lion share of the photon energy of the visible or near-infrared pump light is idle with respect to the lightspin interaction, and the dissipation of the large excess energy represents a major challenge.

In contrast, electromagnetic pulses in the terahertz spectral range $\left(1 \mathrm{THz}=10^{12} \mathrm{~Hz}\right)$ have accessed the spin degree of freedom most directly: The $\mathrm{THz}$ magnetic field exerts a Zeeman torque on the magnetic dipole associated with each spin, at a frequency that may be tuned in resonance with the collective magnon mode [1,3,18-21]. This interaction couples selectively to the spin, without involving charge or lattice degrees of freedom. In materials featuring multiferroic order, spin manipulation has also been mediated by the electric field component of $\mathrm{THz}$ pulses [22]. Conversely, magnetic $\mathrm{THz}$ control is a universal concept, not restricted to multiferroics. It has, indeed, first been demonstrated in an antiferromagnet, representing the largest class of magnetically ordered matter [3]. Since magnetic dipole coupling is typically weak, however, THz-driven spin excitation has been confined to the linear magneto-optical response regime.
Future applications in magnetic storage or quantum computation require a distinctly nonlinear response.

Here, we employ intense $\mathrm{THz}$ pulses with peak magnetic amplitudes of up to $0.4 \mathrm{~T}$ to promote all-magnetic $\mathrm{THz}-$ driven spin dynamics in nickel oxide $(\mathrm{NiO})$ into a new regime characterized by a distinctly nonlinear magneto-optical response. Beside a broadband quasi-instantaneous nonlinearity, an unexpected coherent signature occurs at twice the frequency of the $1 \mathrm{THz}$ antiferromagnetic magnon oscillation. We show that this dynamics is a fingerprint of magnetooptical effects of second order, directly induced by the coherent spin motion. These processes are too fast for magnetostriction to follow and, thus, differ from their static counterparts. Hence they allow us to isolate electron- from lattice-mediated contributions to magnetic linear birefringence (MLB) and dichroism (MLD) - new physics exclusively accessible by strong ultrashort $\mathrm{THz}$ fields.

Below the Néel temperature of $\mathrm{NiO}$ of $523 \mathrm{~K}$, the spins (quantum number $S=1$ ) of the $\mathrm{Ni}^{2+}$ ions order ferromagnetically in equivalent $\{111\}$ planes, whereas adjacent planes are antiferromagnetically exchange-coupled. The four equivalent $\langle 111\rangle$ stacking directions define $T$ domains, wherein the spins point into equivalent $\langle 11 \overline{2}\rangle$ directions, defining three possible $S$ domains for each $T$ domain [3]. The spin system supports well-defined long-wavelength collective magnon modes at frequencies of $\nu=0.23 \mathrm{THz}$ and $1.0 \mathrm{THz}$ at room temperature, corresponding to spin deflection within and out of the $\{111\}$ plane, respectively $[1,3,8,10,12,13]$. Annealing $\mathrm{NiO}$ in an oxygen atmosphere leads to the formation of macroscopic $T$ domains (typical diameter, 10 to $100 \mu \mathrm{m}$ ) containing sub- $\mu \mathrm{m}$-sized $S$ domains. Strong MLB due to magnetostriction along 


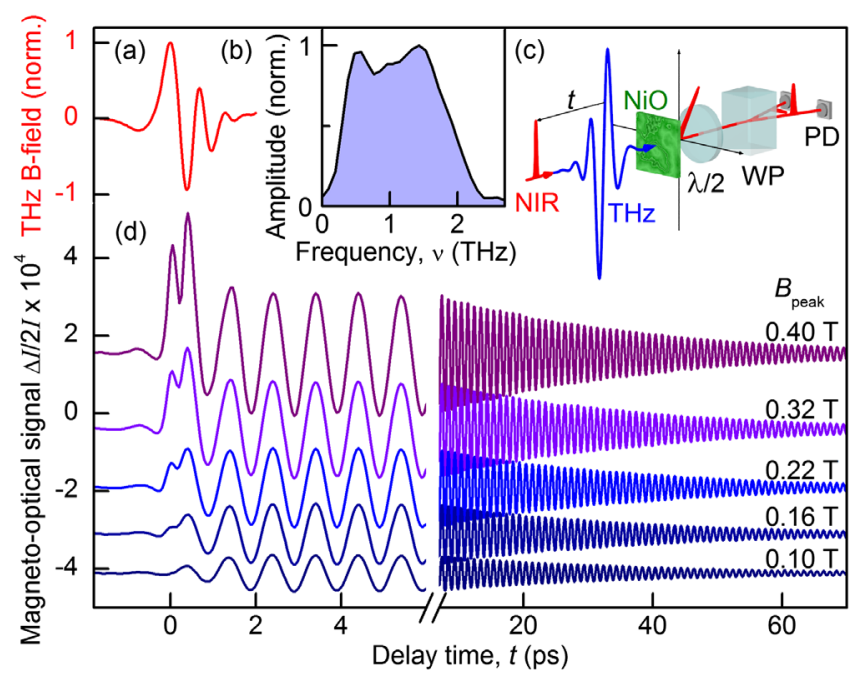

FIG. 1. (a) Waveform of the single-cycle $\mathrm{THz}$ transients used in our experiments. (b) Normalized amplitude spectrum of the $\mathrm{THz}$ transient in (a). (c) Schematic experimental setup: A THz pump (blue) and a copropagating near infrared probe pulse (red) with adjustable temporal delay $t$ are focused onto a free-standing $\mathrm{NiO}$ window (green). The $\mathrm{THz}$ magnetic field induces magnon oscillations by the Zeeman interaction. Because of magnetooptical effects, the transient spin response imprints dynamic polarization modulations on the near infrared probe pulse. A combination of a half-wave plate $(\lambda / 2)$, a Wollaston prism (WP), and two photodiodes (PD) decodes the magneto-optical signal $\Delta I / 2 I$. (d) Magnon oscillations induced by various peak magnetic fields $B_{\text {peak }}$ (right column).

the $\langle 111\rangle$ direction facilitates reliable imaging of the $T$ domains by optical microscopy $[3,8,10,12,13]$.

We generate single-cycle $\mathrm{THz}$ transients [Fig. 1(a)] covering a spectral range from 0.3 to $2 \mathrm{THz}$ (FWHM) [Fig. 1(b)] by tilted-pulse-front optical rectification of near-infrared pulses from a Ti:sapphire laser amplifier (pulse energy, $4 \mathrm{~mJ}$; repetition rate, $3 \mathrm{kHz}$ ) [1]. Using two wire-grid polarizers, the peak magnetic amplitude $B_{\text {peak }}$ may be tuned from 0.05 to $0.4 \mathrm{~T}$, without modifying the waveform of the transients. When focused into a $\langle 111\rangle$-cut $\mathrm{NiO}$ window with a thickness of $45 \mu \mathrm{m}$ [Fig. 1(c)], the THz magnetic field exerts a Zeeman torque on the spins and induces coherent magnon oscillations [3]. The associated transient magneto-optical effects (Faraday effect, MLB, and MLD) are directly captured in the time domain with absolute phase and amplitude by changes in the polarization state of copropagating probe pulses (duration, $30 \mathrm{fs}$; center wavelength, $800 \mathrm{~nm}$; focal diameter FWHM, $5 \mu \mathrm{m}$ ). A half-wave plate and a Wollaston prism translate the THzinduced polarization changes to an imbalance $\Delta I$ of the photocurrents of two identical photodiodes. The magnetooptical signal $\Delta I / 2 I$ ( $I$ denotes the sum of the photocurrents of both diodes) is recorded as a function of the delay time $t$ between the THz pump pulse and the optical probe.

Figure 1(d) displays the temporal traces of the THz-induced magneto-optical signal observed in a single macroscopic $T$ domain, for various field amplitudes. For
$B_{\text {peak }}=0.1 \mathrm{~T}$ (lowest curve) quasimonochromatic magnon oscillations with a period of $1.0 \mathrm{ps}$ build up during the $\mathrm{THz}$ pulse and decay subsequently with a time constant of $\tau=30.5 \mathrm{ps}$. This feature is characteristic of a Faraday signal due to the out-of-plane antiferromagnetic magnon mode of $\mathrm{NiO}$ at $\nu=1.0 \mathrm{THz}$. The excellent stability of our laser system allows us to record the weak signature from a single magnon oscillation period with a signal-to-noise ratio of $\approx 10^{3}$ in an acquisition time of a few seconds. The amplitude of the long-lived monochromatic oscillation scales linearly with $B_{\text {peak }}$ [Fig. 2(a)]. This direct proportionality proves the Zeeman origin of magnon excitation via the magnetic component of the $\mathrm{THz}$ field since linear magneto-electric effects are parity-forbidden in centrosymmetric $\mathrm{NiO}$ [3]. From the size of $\Delta I / 2 I$, we extract a maximum spin deflection angle of $2^{\circ}$ for $B_{\text {peak }}=0.4 \mathrm{~T}$ [3].

In addition to this resonant magnon signature, increasing the peak $\mathrm{THz}$ fields induces a nonlinearity of the magnetooptical response, manifesting itself as two maxima of $\Delta I / 2 I$ at $t=0$ and $0.4 \mathrm{ps}$, whose amplitudes scale quadratically with the THz field [Fig. 2(b)]. This nonlinear signature occurs solely while the $\mathrm{THz}$ field is present, and its shape traces the square of the THz waveform [Fig. 2(c)]. We assign this quasi-instantaneous $\chi^{(3)}$ nonlinearity to a THz-induced Kerr effect observed in other materials before [28].

Whereas this nonresonant nonlinearity is not directly connected with the coherent spin motion, the strong $\mathrm{THz}$ field may also induce a specific nonlinear response due to the spin system, leading to a long-lived magnon response. In order to reveal this aspect, we perform a Fourier transform of $\Delta I / 2 I$ recorded for $B_{\text {peak }}=0.4 \mathrm{~T}$, for delay
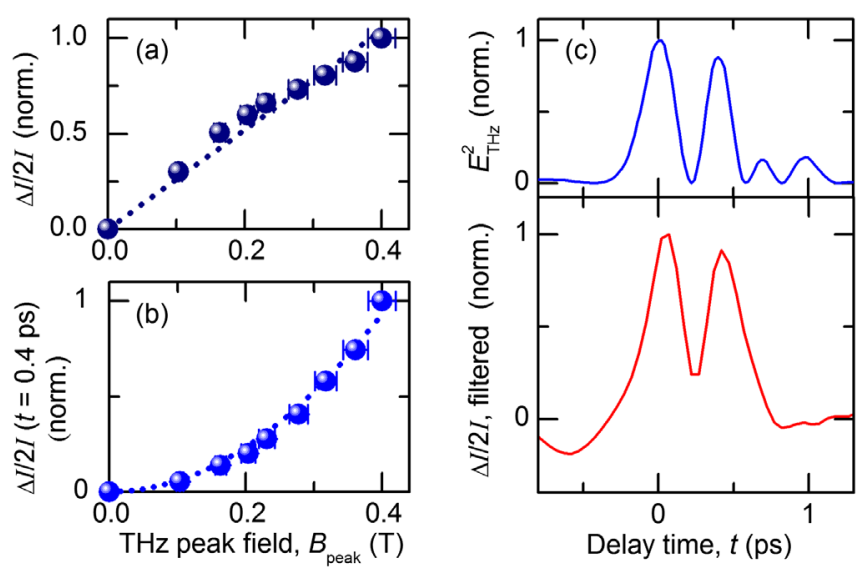

FIG. 2. (a) Normalized amplitude of the magneto-optical signal $\Delta I / 2 I$ of the oscillating signal corresponding to the resonant $1 \mathrm{THz}$ magnon mode (blue spheres, experimental data; dotted line, linear fit) as a function of $B_{\text {peak }}$. (b) Scaling of the peak magneto-optical signal (blue spheres, experimental data; dotted line, quadratic fit) at $t=0.4 \mathrm{ps}$ with respect to $B_{\text {peak }}$. (c) Square of the $\mathrm{THz}$ waveform (blue) and band-block-filtered (stop band, 0.88 to $1.12 \mathrm{THz}$ ) magneto-optical signal (red, $B_{\text {peak }}=0.4 \mathrm{~T}$ ) vs delay time $t$. 
times $t>4 \mathrm{ps}$, i.e., after the instantaneous interaction with the THz field. The spectrum [Fig. 3(a)] is dominated by a Lorentzian peak centered at $\nu=1.0 \mathrm{THz}$, as expected for the Faraday signal due to the out-of-plane magnon mode. The spectral width (FWHM) of $37 \mathrm{GHz}$ is limited by the finite temporal window scanned in the experiment. Intriguingly, an additional maximum is found at $\nu=2.0 \mathrm{THz}$, which corresponds to a polarization modulation at the second harmonic $(\mathrm{SH})$ of the magnon frequency. Its amplitude amounts to $1.4 \%$ of the peak at $1 \mathrm{THz}$ and is consistently observed in all experimental runs with $B_{\text {peak }}=0.4 \mathrm{~T}$. The spectral weight of the SH signature (see [29], part 1 for details of the analysis) scales quadratically with the $\mathrm{THz}$ peak field [inset to Fig. 3(a)]. This finding marks the first $\mathrm{THz}$ induced response at a harmonic frequency of a magnetic resonance, to the best of our knowledge. The shape of the SH spectral signature depends sensitively on the probe polarization [Fig. 3(b)]: When the polarization of the THz pump and the position of the crystal is kept fixed while we rotate the polarization of the probe pulse, the $\mathrm{SH}$ feature morphs from a peak into a dip and back into a peak with $180^{\circ}$ period.

While the nonlinear response of spins in ferrimagnetic materials in the $\mathrm{GHz}$ spectral range has been extensively
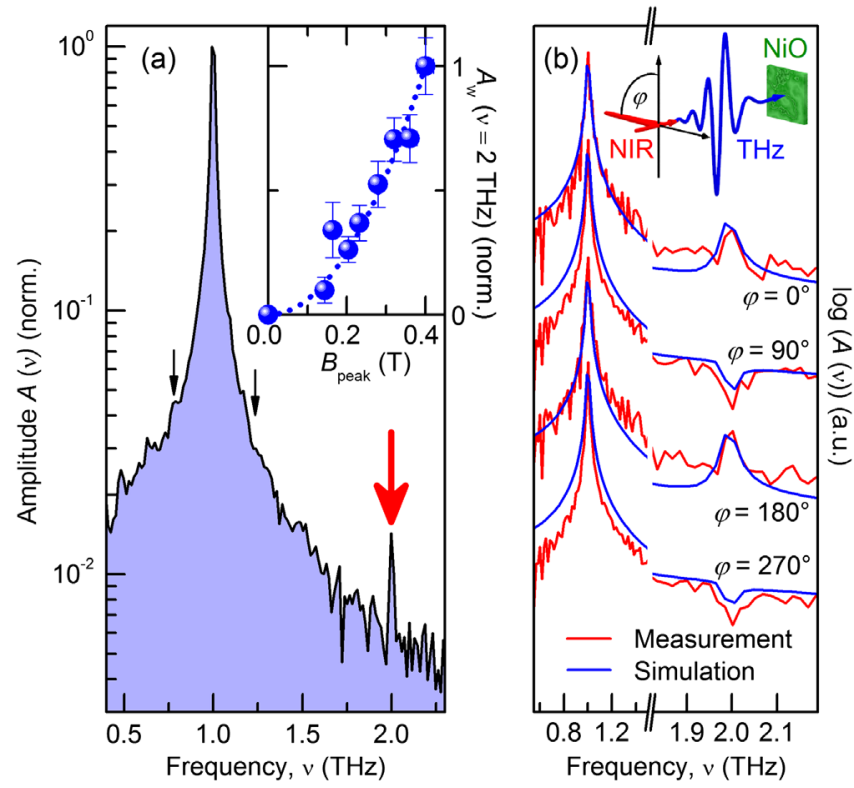

FIG. 3. (a) Normalized spectrum of the magneto-optical signal for $B_{\text {peak }}=0.4 \mathrm{~T}$ [see Fig. 1(d), top curve] extracted for delay times $t>4 \mathrm{ps}$. The sharp Lorentzian peak at $1 \mathrm{THz}$ results from the long-lived oscillation of the $1-\mathrm{THz}$ magnon mode. Surprisingly, an additional peak is found at the second harmonic frequency $\nu=2.0 \mathrm{THz}$. Black arrows: spectral positions of sum- and difference-frequency components of the 1 and $0.23-\mathrm{THz}$ magnon mode. Inset: Spectral weight $A_{w}$ of the peak at $\nu=2.0 \mathrm{THz}$ as a function of the THz driving field $B_{\text {peak }}$ (blue spheres, experimental data; dotted line, quadratic fit). (b) Magnon spectra induced by THz peak fields of $0.4 \mathrm{~T}$ for various angles $\varphi$ of the probe polarization with respect to the crystal lattice (red curves, experimental data; blue curves, simulation). studied [37], the observed SH response in a pure antiferromagnet comes as a surprise. In order to explain this fact, we first analyze the spin motion itself and demonstrate that the corresponding Faraday signal cannot account for the $\mathrm{SH}$ signature. For small spin deflection angles, the transient magnetization associated with the coherent magnon oscillates harmonically and nonlinearities of the Faraday signal are obviously excluded. For larger magnon amplitudes, one has to account for the fact that a single electron spin is a prototypical saturable quantum system with an inherently nonlinear response. Yet still in this case, we can show that nonlinearities of the transient magnetization at the $\mathrm{SH}$ are expected to cancel exactly in a perfect antiferromagnet. To this end, we model the THz-induced spin dynamics in $\mathrm{NiO}$ microscopically, following the theory of Refs. [3] and [38], which describes the collective long-wavelength magnon mode as a uniform precession of all spins located on the two antiferromagnetic sublattices. The model includes exchange coupling, anisotropy, and direct Zeeman coupling of the spins with the measured $\mathrm{THz}$ waveform. We analyze the semiclassical three-dimensional trajectories of the two spin vectors $\mathbf{S}_{1}$ and $\mathbf{S}_{2}$ representing the antiferromagnetic sublattices on the Bloch sphere and identify the frequency components supported by the system.

Following THz excitation, $\mathbf{S}_{1}$ and $\mathbf{S}_{2}$ precess about their equilibrium direction ( $z$ axis) on the Bloch sphere [Fig. 4(a)]. Viewing along the $z$ axis, the spins rotate in opposite directions and undergo different deflections within the $\{111\}$ plane (containing the $y$ and $z$ axes) and out-ofplane ( $x$ axis). The Cartesian $x$ components of $\mathbf{S}_{1}$ and $\mathbf{S}_{2}$ are $180^{\circ}$ out of phase with respect to each other [Fig. 4(b)] whereas the $y$ components are in phase, due to the action of the anisotropy field. Interestingly, the elliptical trajectories on the Bloch sphere change the $z$ component of both spins
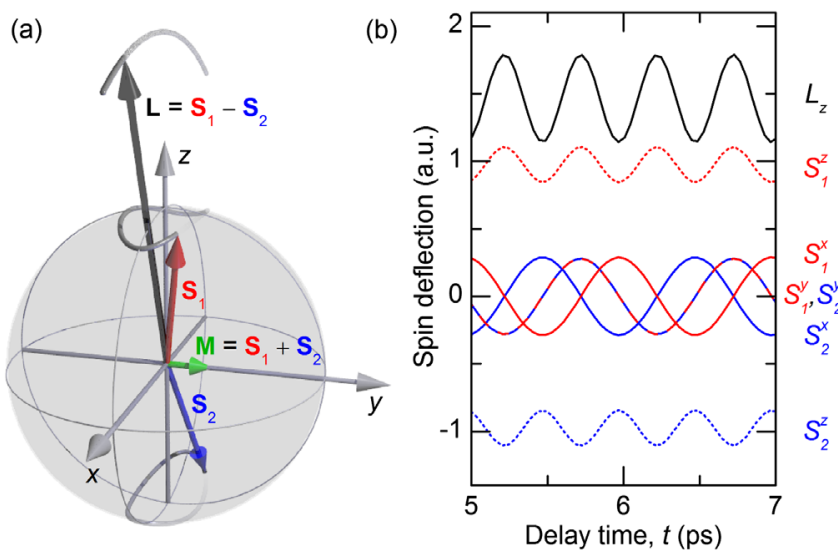

FIG. 4. (a) Bloch sphere representation of typical trajectories of the spins $\mathbf{S}_{1}$ and $\mathbf{S}_{2}$ in the two antiferromagnetic spin sublattices. While the transient magnetization $\mathbf{M}=\mathbf{S}_{1}+\mathbf{S}_{2}$ cancels along $x$ and $z$, the anisotropy field causes a nonzero net magnetization along $y$. The antiferromagnetic vector $\mathbf{L}=\mathbf{S}_{1}-\mathbf{S}_{2}$ oscillates in the $x-z$ plane. (b) Cartesian components of $\mathbf{S}_{1}$ and $\mathbf{S}_{2}$ and the Cartesian component $L_{z}$ of the antiferromagnetic vector $\mathbf{L}$ for a schematic motion as shown in panel (a). 
at the $\mathrm{SH}$ frequency. This frequency component, however, does not contribute to the total magnetization $\mathbf{M} \propto \mathbf{S}_{1}+\mathbf{S}_{2}$ because the $z$ coordinates of both spins cancel [Fig. 4(a), green arrow]. The Faraday signal, which is proportional to $\mathbf{M}$, is expected to reflect only the 1-THz oscillation along $y$. This is also fully supported by the established analytical theory of nonlinear dynamics in antiferromagnets (see [29], part 2). In a perfect antiferromagnetic crystal, this observation holds even for large spin deflections, i.e., for strong $\mathrm{THz}$ peak fields. Because the cancellation of SH signatures in $\mathbf{M}$ occurs within a single unit cell, it applies equally to all $S$ and $T$ domains. Accordingly, the amplitude spectrum of the transient magnetization $\mathbf{M}$ computed for $B_{\text {peak }}=0.4 \mathrm{~T}$ (see [29], Fig. S1) is dominated by the fundamental peak at $\nu=1.0 \mathrm{THz}$ whereas a $\mathrm{SH}$ response is absent. Local maxima at $\nu=1.23$ and $0.77 \mathrm{THz}$ can be assigned to difference- and sum-frequency mixing of the in-plane and out-of-plane magnon oscillation. Our experimental spectra actually exhibit faint shoulders at these spectral positions [Fig. 3(a), black arrows], but the finite width of the fundamental magnon peak prevents a clear isolation of these maxima.

The $\mathbf{S H}$ component of either $\mathbf{S}_{1}$ or $\mathbf{S}_{2}$ can only cause a Faraday response if the two sublattices are not perfectly compensated, e.g., due to domain boundaries, crystal defects, or strain. Such effects, however, would be independent of the probe polarization and are thus inconsistent with the symmetry of the response observed in Fig. 3(b) (see [29], part 3). Similarly, our polarization analysis excludes a transient breaking of the antiferromagnetic symmetry by the $\mathrm{THz}$ pump pulse as a potential origin of the SH oscillation, since such symmetry breaking would be the same for all probe polarizations (see [29], part 2, for a theoretical analysis).

Finally, we note that for very large spin deflection amplitudes the magnon frequency itself may become amplitude dependent. In a damped oscillation, one would then expect a time dependence of the instantaneous magnon frequency, which may cause intriguing nonlinearities [39]. Such effects, however, are not seen in our experiment. The above considerations lead us to the conclusion that spin dynamics probed by Faraday rotation (scaling linearly with $\mathbf{M}$ ) cannot be the origin of the $\mathrm{SH}$ signal in our experiment.

Consequently, we turn towards possible sources of the SH frequency caused by the probe process itself. First, the oscillating magnetization probed by the Faraday effect may cause a series of harmonic sidebands as observed in phonon-modulated silicon [40]. However, this effect only leads to odd multiples of the magnon frequency (see [29], part 2, especially Eq. (9), for an exact calculation). Second, the interplay between a periodic $1-\mathrm{THz}$ modulation of the complex refractive index and the static birefringence of $\mathrm{NiO}$ may cause propagation effects. This is accounted for by a full quantitative model (see [29], part 4), which shows that propagation effects in the crystal are 2 orders of magnitude too small in amplitude to explain the experimental findings of the SH signature ([29], Fig. S1).

Instead, we now show that the strong spin deflection allows us to access transient magneto-optical effects of second order in the antiferromagnetic vector $\mathbf{L}=\mathbf{S}_{1}-\mathbf{S}_{2}$ that are contained in the diagonal elements of the dielectric permittivity tensor $[41,42]$. Their real and imaginary parts induce a difference in the refractive indices (MLB) and absorption coefficients (MLD), respectively, along and perpendicularly to $\mathbf{L}$. In particular, the terms $L_{x}^{2}$ and $L_{z}^{2}=$ $L_{0}^{2}-L_{x}^{2} \approx L_{0} d L$ directly probe the coherent [43] nonlinear magnon response functions, where $L_{0}$ is the equilibrium value of $|\mathbf{L}|$ (see [29], part 5) and $d L$ is the dynamic component of $L_{z}$. The transient birefringence and dichroism are thus expected to oscillate at the $\mathrm{SH}$ frequency (Fig. 4) and to scale quadratically with the $\mathrm{THz}$ fields, as seen in the experiment [Fig. 3(a)], while the spin deflection angle [Fig. 2(a)] remains in the linear regime. Analogously to static magnetic linear birefringence and dichroism, transient MLB and MLD are expected to be anisotropic with respect to the probe polarization [42].

To confirm our interpretation, we extended the propagation model used above by introducing a complex Jones matrix including both MLB and MLD (difference of ordinary and extraordinary refractive indices or absorption coefficients for the transient dynamics). As seen in Fig. 3(b) (blue line), our model describes the local maximum of the magneto-optical signal at $\nu=2 \mathrm{THz}$ and reproduces the change of the local maximum into a local minimum as the probe polarization is rotated by $90^{\circ}$. This peculiarity is caused by a phase-sensitive superposition of the polarization effect of the transient MLB and MLD at the SH and the broad Lorentzian Faraday signature centered at $1 \mathrm{THz}$. As the probe polarization is rotated, the relative phase between both effects changes, alternating between constructive and destructive interference.

Note that the subcycle time resolution of the MLB and MLD [44] warrants access to a mechanism that is fundamentally different from static birefringence or dichroism. The latter is dominated by magnetostriction $[3,8,10,12,13]$. In contrast, the associated elastic lattice deformation cannot follow the ultrafast $\mathrm{THz}$ spin precession studied here. Therefore, the transient second-order magneto-optical effects are directly related to the dependence of the electronic structure on the spin deflections and much weaker than their static counterparts. Our experimental data are well reproduced with a maximum phase difference between the ordinary and extraordinary waves on the order of $\mu \mathrm{rad}$, whereas static MLB induces phase differences on the order of $1 \mathrm{rad}$. The dynamic absorption coefficient due to MLD lies on the order of $10^{-4} \mathrm{~cm}^{-1}$ to account for the experimental results, whereas its static counterpart amounts to $\sim 400 \mathrm{~cm}^{-1}$ [45]. In future studies, the contributions to the nonlinear SH signal due to MLB and MLD may even be precisely adjusted by tuning the probe wavelength. 
Since the novel nonlinearity is not obscured by lattice dynamics it opens up an elegant way to complement Faraday measurements. While Faraday rotation probes the transverse spin deflection, the transient MLB and MLD simultaneously explore the motion of the longitudinal spin component on a subcycle time scale. Such information will be particularly important when future $\mathrm{THz}$ magnetic control explores spin switching. We note that with increasing spin deflection angles additional spectral components of the transient MLB and MLD are expected at frequencies of 1, 3, and $4 \mathrm{THz}$ (see [29], part 5). Such signatures may serve as valuable indicators for future nonperturbative spin dynamics.

In conclusion, we demonstrated an all-magnetic $\mathrm{THz}-$ driven nonlinear optical spin response. Beside an instantaneous $\chi^{(3)}$ nonlinearity and indications of mixing of two antiferromagnetic modes, a distinct $\mathrm{SH}$ signal of the fundamental magnon oscillation, scaling quadratically with the $\mathrm{THz}$ amplitude, is found in $\mathrm{NiO}$. We demonstrate that this signature is at odds with linear Faraday rotation induced by large-amplitude magnon oscillations. The novel nonlinearity is well explained by ultrafast magneto-optical effects scaling quadratically with the longitudinal spin component. Our results extend the established Faraday measurements of transverse spin deflection to coherent longitudinal spin dynamics. While static MLB has been shown to be mediated by magnetostriction (i.e., a lattice distortion proportional to $\mathbf{L}^{2}$ ), this coupling mechanism cannot explain the dynamic signature seen at $2 \mathrm{THz}$. Since there are no long-wavelength acoustic phonons at $2 \mathrm{THz}$, the crystal lattice cannot adiabatically follow the $\mathrm{THz}$ spin precession. Hence our approach allows us to dynamically separate electronic from magnetostrictive contributions to coherent magnon-induced birefringence. We anticipate that these signatures will blaze the trail towards all-magnetic $\mathrm{THz}$ spin switching.

We thank Martin Furthmeier for technical assistance and Rostislav Mikhaylovskiy, Ulrich Nowak and Davide Bossini for fruitful discussions. This work was supported by the European Research Council through Grant No. 305003 (QUANTUMsubCYCLE) and Grant No. 681917 (TERAMAG), by the Deutsche Forschungsgemeinschaft (DFG) through Collaborative Research Center (Sonderforschungsbereich) SFB 689 and Priority Program (Schwerpunktprogramm) SPP 1666, and by the Nederlandse Organisatie voor Wetenschappelijk Onderzoek (NWO) through a Rubicon grant and a VENI grant.

*upert.huber@physik.uni-regensburg.de

[1] T. Kampfrath, K. Tanaka, and K. Nelson, Nat. Photonics 7, 680 (2013).

[2] A. Kirilyuk, A. V. Kimel, and T. Rasing, Rev. Mod. Phys. 82, 2731 (2010).
[3] T. Kampfrath, A. Sell, G. Klatt, A. Pashkin, S. Maehrlein, T. Dekorsy, and R. Huber, Nat. Photonics 5, 31 (2011).

[4] T. Satoh, R. Iida, T. Higuchi, M. Fiebig, and T. Shimura, Nat. Photonics 9, 25 (2015).

[5] V. Kimel, A. Kirilyuk, P. A. Usachev, R. V. Pisarev, A. M. Balbashov, and T. Rasing, Nature (London) 435, 655 (2005).

[6] V. Kimel, A. Kirilyuk, A. Tsvetkov, R. V. Pisarev, and T. Rasing, Nature (London) 429, 850 (2004).

[7] T. Satoh, Y. Terui, R. Moriya, B. Ivanov, K. Ando, and E. Saitoh, Nat. Photonics 6, 662 (2012).

[8] J. Nishitani, T. Nagashima, and M. Hangyo, Phys. Rev. B 85, 174439 (2012).

[9] Z. Jin, H. Ma, L. Wang, G. Ma, F. Guo, and J. Chen, Appl. Phys. Lett. 96, 201108 (2010).

[10] T. Satoh, S.-J. Cho, R. Iida, T. Shimura, K. Kuroda, H. Ueda, Y. Ueda, B. A. Ivanov, F. Nori, and M. Fiebig, Phys. Rev. Lett. 105, 077402 (2010).

[11] F. Hansteen, A. Kimel, A. Kirilyuk, and T. Rasing, Phys. Rev. Lett. 95, 047402 (2005).

[12] N. Kanda, T. Higuchi, H. Shimizu, K. Konishi, K. Yoshioka, and M. Kuwata-Gonokami, Nat. Commun. 2, 362 (2011).

[13] T. Higuchi, N. Kanda, H. Tamaru, and M. KuwataGonokami, Phys. Rev. Lett. 106, 047401 (2011).

[14] V. Kimel, B. A. Ivanov, R. V. Pisarev, P. A. Usachev, A. Kirilyuk, and T. Rasing, Nat. Phys. 5, 727 (2009).

[15] R. V. Mikhaylovskiy et al., Nat. Commun. 6, 8190 (2015).

[16] J. H. Mentink, K. Balzer, and M. Eckstein, Nat. Commun. 6, 6708 (2015).

[17] J. H. Mentink and M. Eckstein, Phys. Rev. Lett. 113, 057201 (2014).

[18] Z. Jin, Z. Mics, G. Ma, Z. Cheng, M. Bonn, and D. Turchinovich, Phys. Rev. B 87, 094422 (2013).

[19] R. Zhou, Z. Jin, G. Li, G. Ma, Z. Cheng, and X. Wang, Appl. Phys. Lett. 100, 061102 (2012).

[20] M. Nakajima, A. Namai, S. Ohkoshi, and T. Suemoto, Opt. Express 18, 18260 (2010).

[21] K. Yamaguchi, M. Nakajima, and T. Suemoto, Phys. Rev. Lett. 105, 237201 (2010).

[22] T. Kubacka et al., Science 343, 1333 (2014).

[23] K. W. Kim, A. Pashkin, H. Schäfer, M. Beyer, M. Porer, T. Wolf, C. Bernhard, J. Demsar, R. Huber, and A. Leitenstorfer, Nat. Mater. 11, 497 (2012).

[24] Z. Wang, M. Pietz, J. Walowski, A. Förster, M. I. Lepsa, and M. Münzenberg, J. Appl. Phys. 103, 123905 (2008).

[25] C. H. Back, R. Allenspach, W. Weber, S. S. P. Parkin, D. Weller, E. L. Garwin, and H. C. Siegmann, Science 285, 864 (1999).

[26] S. Takahashi, L.-C. Brunel, D. T. Edwards, J. van Tol, G. Ramian, S. Han, and M. S. Sherwin, Nature (London) 489, 409 (2012).

[27] T. Kampfrath et al., Nat. Nanotechnol. 8, 256 (2013).

[28] M. C. Hoffmann, N. C. Brandt, H. Y. Hwang, K.-L. Yeh, and K. A. Nelson, Appl. Phys. Lett. 95, 231105 (2009).

[29] See Supplemental Material http://link.aps.org/supplemental/ 10.1103/PhysRevLett.117.197201, which includes Refs. [30-36].

[30] A. F. Andreev and V. I. Marchenko, Usp. Fiz. Nauk 130, 39 (1980) [Phys. Usp. 23, 21 (1980)]. 
[31] V. G. Baryakhtar, B. A. Ivanov, and A. L. Sukstanskii, Zh. Eksp. Teor. Fiz. 78, 1509 (1980) [Sov. Phys. JETP 51, 757 (1980)].

[32] B. A. Ivanov and A. Sukstanskii, Zh. Eksp. Teor. Fiz. 84, 370 (1983) [Sov. Phys. JETP 57, 214 (1983)].

[33] V. G. Baryakhtar and A. G. Danilevich, Low Temp. Phys. 39, 993 (2013).

[34] S. Sugano and N. Kojima, Magneto-Optics (Springer, Berlin, 2000), Vol. 128, pp. 137-145.

[35] S. Huard, Polarization of Light (John Wiley \& Sons, Chichester, 1996).

[36] H. Kondoh and T. Takeda, J. Phys. Soc. Jpn. 19, 2041 (1964).

[37] M. G. Cottam, Linear and Nonlinear Spin Waves in Magnetic Films and Superlattices (World Scientific, Singapore, 1994).
[38] S. Wienholdt, D. Hinzke, and U. Nowak, Phys. Rev. Lett. 108, 247207 (2012).

[39] Y. Mukai, H. Hirori, T. Yamamoto, H. Kageyama, and K. Tanaka, New J. Phys. 18, 013045 (2016).

[40] M. Hase, M. Katsuragawa, A. M. Constantinescu, and H. Petek, Nat. Photonics 6, 243 (2012).

[41] D. Bossini, A. M. Kalashnikova, R. V. Pisarev, T. Rasing, and A. V. Kimel, Phys. Rev. B 89, 060405 (2014).

[42] J. Ferre and G. A. Gehring, Rep. Prog. Phys. 47, 513 (1984).

[43] We note that MLB was used in [41] to probe incoherent magnetization dynamics due to laser heating of the magnon system.

[44] The NiO crystal is absorptive and birefringent [45]. Because of the Kramers-Kronig relations, MLB in the presence of absorption must lead to MLD.

[45] R. Newman and R. M. Chrenko, Phys. Rev. 114, 1507 (1959). 